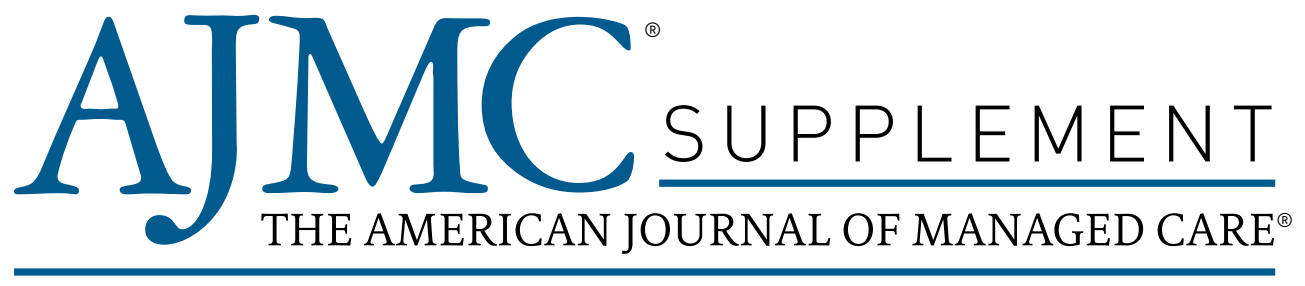

November 2020 Vol. 26 • No. 15 , Sup.

\title{
Economic Implications of Osteoporotic Fractures in Postmenopausal Women
}

\section{HIGHLIGHTS}

> Economic Implications of Osteoporotic Fractures in Postmenopausal Women

> CE Sample Posttest 


\section{Economic Implications of Osteoporotic Fractures in Postmenopausal Women}

Release date: November 30, 2020

Expiration date: November 30, 2021

Estimated time to complete activity: 1.0 hour

Type of activity: Application

Medium: Internet-based posttest, evaluation, and request for credit

Fee: Free

This activity is supported by an educational grant from Radius Health, Inc.

\section{Intended Audience}

Managed care payers, pharmacy directors, pharmacy benefit managers, specialty pharmacy directors, and any other pharmacist and/or healthcare professional interested in scientific advances in the treatment of osteoporosis.

\section{Activity Overview}

Osteoporosis is a preventable and treatable disease that most commonly occurs in postmenopausal women. However, its primary symptom of bone fragility is often not identified until there has been a trauma, such as fracture from a fall. Pharmacists must be familiar with the patient-specific risk factors associated with development of osteoporosis and can educate about prevention strategies, such as early and diagnostic screening, and provide nonpharmacologic recommendations for optimal vitamin D and calcium supplementation. This article will review several pharmacologic therapies used for the prevention and treatment of osteoporosis that pharmacists need to be familiar with to support reduction in fracture risk for their patients. Equipped with knowledge of these prevention and treatment strategies, pharmacists can be integral to developing prior authorization and step therapy processes within managed care organizations and ensuring transitions of care after a fracture.

\section{Statement of Educational Need}

While the treatment landscape for osteoporosis has grown in recent years, there are still significant unmet needs in care. Almost $50 \%$ of all osteoporotic hip fractures could be avoided with appropriate preventive strategies. Managed care pharmacists play a role in filling these gaps in care. Continuing education will improve managed care pharmacists' understanding of the risks of osteoporosis and osteoporotic fractures in postmenopausal women, insight into emerging treatments, and strategies to create access to these novel therapies.

\section{Educational Objectives}

Upon completion of this activity, participants will be able to:

- Investigate the risks of osteoporosis and osteoporotic fractures in postmenopausal women.

- Examine clinical data for emerging therapies for the treatment of osteoporosis in postmenopausal women.

- Explore the role of managed care professionals in creating access to novel therapies for patients at risk for osteoporosis or for osteoporotic fractures.

\section{Accreditation Statement}

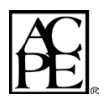
Pharmacy Times Continuing Education ${ }^{\mathrm{TM}}$ is accredited by the Accreditation Council for Pharmacy Education (ACPE) as a provider of continuing pharmacy education. This activity is approved for 1.0 contact hour $(0.10 \mathrm{CEU})$ under the ACPE universal activity number 0290-0000-20-380-H01-P. The activity is available for CE credit through November 30, 2021.

Obtaining Credit: Participants must read the article and complete the online posttest and an online evaluation and request for credit. Detailed instructions on obtaining CE credit are included at the end of this activity.

This CE activity is offered free online at www.ajmc.com/ce and at www. PharmacyTimes.org, where you will be directed to the activity in its entirety, including the online pretest and posttest, activity evaluation, and request for credit. 


\section{SUPPLEMENT}

\section{Economic Implications of Osteoporotic Fractures in Postmenopausal Women}

\begin{abstract}
OVERVIEW
Through this supplement to

The American Journal of

Managed Care ${ }^{\circledR}$, managed

care professionals will

increase their knowledge

of current and emerging

therapies for the prevention of

fractures and osteoporosis in

postmenopausal women.
\end{abstract}

\section{TABLE OF CONTENTS}

Participating Faculty

Report

Economic Implications of Osteoporotic Fractures

in Postmenopausal Women

Jeenal Patel, PharmD, BCGP

CE Sample Posttest

S319 


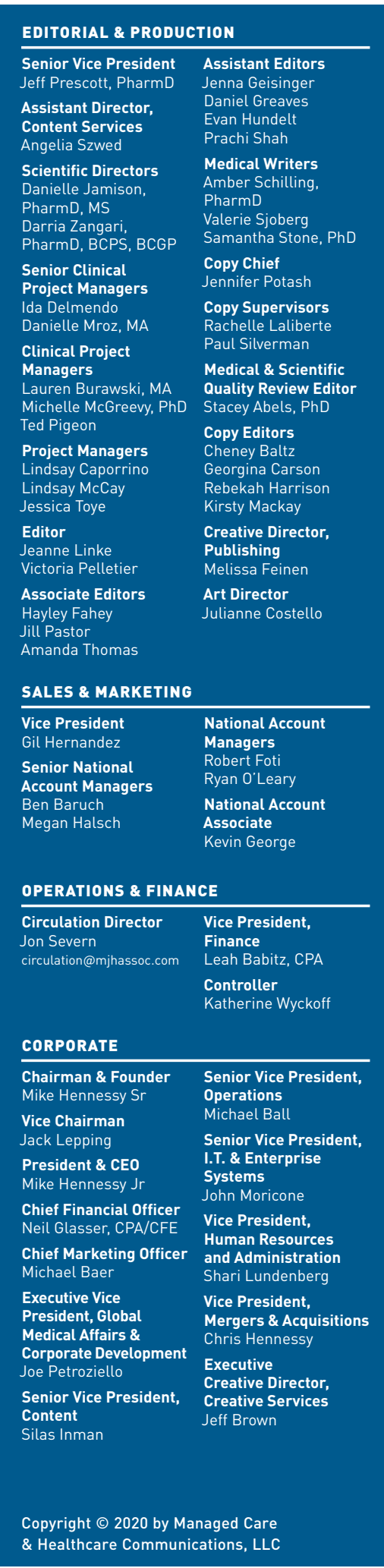

AN N- life sciences" BRAND

\section{FACULTY}

Jeenal Patel, PharmD, BCGP

Formulary Manager

WellDyne

Lakeland, Florida

\section{FACULTY DISCLOSURES}

Jeenal Patel, PharmD, BCGP, has no relevant financial relationships with commercial interests to disclose.

\section{EDITORIAL SUPPORT DISCLOSURES}

Pharmacy Times Continuing Education ${ }^{\mathrm{TM}}$

Planning Staff: Jim Palatine, RPh, MBA; Maryjo Dixon, RPh; Kimberly Simpson, PharmD; Kelly McCormick; Susan Pordon; and Brianna Winters have no relevant financial relationships with commercial interests to disclose.

\section{DISCLOSURE POLICY}

According to the disclosure policy of The American Journal of Managed Care ${ }^{\circledR}$ and Pharmacy Times Continuing Education ${ }^{\mathrm{TM}}$, all persons who are in a position to control content are required to disclose any relevant financial relationships with commercial interests. If a conflict is identified, it is the responsibility of Pharmacy Times Continuing Education ${ }^{T M}$ to initiate a mechanism to resolve the conflict(s). The existence of these relationships is not viewed as implying bias or decreasing the value of the activity. All educational materials are reviewed for fair balance, scientific objectivity of studies reported, and levels of evidence.

\section{DISCLOSURE OF UNAPPROVED/OFF-LABEL USE}

The contents of this activity may include information regarding the use of products that may be inconsistent with or outside the approved labeling for these products in the United States. Participants should note that the use of these products outside current approved labeling is considered experimental and they are advised to consult prescribing information for these products.

The information provided in this CE activity is for continuing medical and pharmacy education purposes only and is not meant to substitute for the independent medical or pharmacy judgment of a physician or pharmacist relative to diagnostic, treatment, or management options for a specific patient's medical condition.

The opinions expressed in the content are solely those of the individual faculty members and do not reflect those of The American Journal of Managed Care ${ }^{\circledR}$, Pharmacy Times Continuing Education ${ }^{\mathrm{TM}}$, or any of the companies that provided commercial support for this CE activity.

Signed disclosures are on file at the office of The American Journal of Managed Care ${ }^{\circledR}$, Cranbury, New Jersey. 


\title{
Economic Implications of Osteoporotic Fractures in Postmenopausal Women
}

\author{
Jeenal Patel, PharmD, BCGP
}

\section{Pathophysiology of Osteoporosis}

Osteoporosis occurs when bone tissue loses its structural matrix, resulting in low bone density and increased skeletal fragility. The microscopic appearance of osteoporotic bone has been well established. Bone analysis has revealed loss of fibrillar form and degradation of collagen contributing to its loss of density and increased fracture risk. ${ }^{1}$ The pathophysiology of osteoporosis involves 2 distinct mechanisms: (1) failure to achieve peak bone mass and (2) disproportional increase in bone resorption during bone remodeling. Peak bone mass is critical for the prevention of osteoporotic bone and subsequent bone fractures. For instance, a $30 \%$ reduction of hip fracture can be achieved by increasing peak bone mass by $10 \%$. Factors that contribute to peak bone mass include genetics, hormonal status (particularly estrogen), nutrition, exercise, and smoking. ${ }^{2}$ Bone remodeling is the process by which osteoclasts (bone resorbing cells) and osteoblasts (bone forming cells) repair bone microdamage and maintain bone health. Estrogen is key hormonal regulator of bone health maintenance. Estrogen inhibits bone resorption, largely by direct actions on osteoclasts. ${ }^{3}$ Estrogen deficiency in menopause causes a disproportionate increase in bone resorption compared with bone formation. This imbalance is one of the major factors in the formation of osteoporotic bone.

\section{Diagnostic Criteria and Contributing Risk Factors}

The National Osteoporosis Foundation (NOF) recommends that all postmenopausal women be evaluated for osteoporotic risk to determine the need for bone mineral density (BMD) testing. ${ }^{4}$ Although osteoporosis is preventable and treatable, the diagnosis is often made after a fracture has occurred. This is mainly due to the lack of symptoms in the early stages of the disease and limited medical care during this time. However, early diagnosis can significantly improve outcomes. The World Health Organization (WHO) established a classification of BMD by dual-energy x-ray absorptiometry (DEXA). Osteoporosis is diagnosed when the T-score, defined by the WHO as the standard deviation difference between a patient's BMD compared with that of a young adult, is less than or equal to -2.5 . Indications for testing include women older than 65 years

\section{ABSTRACT}

Osteoporosis is a condition affecting millions of Americans, the majority of which are women secondary to low estrogen in the postmenopausal state. In many instances, the diagnosis is not recognized until after a fracture has occurred due to the "silent" nature of this disease. As a result, this leads to millions of fractures each year, placing a substantial economic burden of billions of dollars from Medicare alone. Prevention, early diagnosis, and treatment remain paramount in reducing this economic impact as well as improving overall incidence of mortality and morbidity. Goals of care include preventing the progression of disease and minimizing bone fractures, particularly in the hips, spine, and wrists. This article will examine the risks of osteoporosis and osteoporotic fractures in postmenopausal women, the current and emerging pharmacologic treatment options, and the role of managed care pharmacists in decreasing economic burden and improving patient care.

Am J Manag Care. 2020;26:S311-S318

For author information and disclosures, see end of text. 
and younger postmenopausal women with clinical risk factors for fracture (eg, low body weight $<57.6 \mathrm{~kg}$ or a family history of spine or hip fractures). ${ }^{5}$

According to the National Bone Health Alliance (NBHA), osteoporosis in a postmenopausal woman may be diagnosed by one of the following methods ${ }^{6}$ :

- Individual has sustained a low trauma (eg, fall from standing) hip fracture

- Presence of osteopenia or low bone mass confirmed by BMD (T-score of less than -1 but greater than -2.5 ) and individual has experienced a vertebral, proximal humeral, pelvic, or distal forearm fracture

- Using the WHO fracture risk assessment (FRAX) tool 10-year probability indicating hip fracture risk is greater than or equal to $3 \%$ or the 10 -year probability of major osteoporotic fracture risk is greater than or equal to 20

The FRAX tool takes into consideration several risk factors, including current age, rheumatoid arthritis, gender, secondary causes of osteoporosis (eg, type 1 diabetes, osteogenesis imperfecta in adults, untreated long-standing hyperthyroidism, hypogonadism, or premature menopause [ $<40$ years], chronic malnutrition or malabsorption, and chronic liver disease), a prior osteoporotic fracture (including clinical and asymptomatic vertebral fractures), parental history of hip fracture, femoral neck BMD, current smoking, low body mass index, alcohol intake ( $\geq 3$ drinks/day), and oral glucocorticoids greater than or equal to $5 \mathrm{mg}$ /day of prednisone for longer than 3 months. ${ }^{6}$

\section{Epidemiology and Consequences}

An estimated 10.2 million individuals in the United States have osteoporosis and $80 \%$ are women. ${ }^{7}$ This imbalance is secondary to the significant bone loss in the postmenopausal state. In addition to the 10.2 million people with osteoporosis, another 43.4 million have osteopenia, a state that, if left untreated, will lead to osteoporosis and subsequent fractures. Every year, 2 million fractures due to osteoporosis occur in the United States and 1 of 2 women older than 50 years will have an osteoporosis-related fracture during her lifetime. ${ }^{8}$ Medicare currently pays for most of these costs, insuring a substantial economic burden. This cost is expected to increase to $\$ 25$ billion by 2025 as the population ages. In postmenopausal women, osteoporotic fracture costs exceed the annual costs for breast cancer, myocardial infarction (MI), and stroke. Although largely treatable, fewer than $25 \%$ of women older than 67 years with an osteoporotic fracture undergo treatment or obtain a BMD measurement. ${ }^{9}$

\section{Prevention of Fractures and Osteoporosis: Current and Emerging Therapies}

According to the NOF, there is a large unmet need as it relates to the prevention of postmenopausal osteoporosis because many women are not undergoing the recommended diagnostic screenings or assessing baseline osteoporosis risk. ${ }^{4}$ Additionally, there is evidence of low BMD screening rates by healthcare providers. ${ }^{10}$ Both nonpharmacologic and pharmacologic therapies are used for the prevention and treatment of osteoporosis.

Many well established organizations, including the NOF, have developed guidance on osteoporosis treatment. Others include:

- The American Association of Clinical Endocrinologists and American College of Endocrinology (AACE/ACE)

- The Endocrine Society (ES)

- The North American Menopause Society (NAMS)

- The American College of Rheumatology (ACR)

The goals of osteoporosis treatment are to control the progression of the disease and minimize the risk of bone fractures, particularly in the hips, spine, and wrists, and prevent falls and fractures. ${ }^{11}$ The decision to continue treatment or switch therapies may be contingent upon changes in BMD, bone turnover markers, and the occurrence of fractures. Choice of therapy is based on a combination of factors, including efficacy, safety, overall treatment cost, and patient characteristics such as fracture risk, other health comorbidities, and medications. Several generic and brand options exist for treatment and are generally recommended for patients diagnosed with osteoporosis. At the minimum, patients should regularly perform weight-bearing and muscle-strengthening exercises for at least 30 minutes on 3 or 4 days per week to help reduce the risk of falls and improve balance and flexibility. Lifestyle modifications, such as limiting caffeine intake, ensuring adequate protein in daily diet, and avoiding smoking and excessive alcohol intake, can help reduce the negative impact on the skeleton. ${ }^{11}$ There are varying recommendations for optimal vitamin $\mathrm{D}$ and calcium supplementation. The NOF recommends 800-1000 units of vitamin D per day in women aged 50 years and older and $1200 \mathrm{mg}$ of calcium accumulated from both diet and supplements for women aged 51 years and older. Taking fall prevention measures around the home can be key to reducing fracture risk. Patients can wear rubber soles for better grip, use devices to assist with walking, such as a cane or walker, remove loose electrical wires and cords, and use handrails when possible.

\section{Bone Resorptive Agents}

\section{Bisphosphonates}

Bisphosphonates are generally recommended as first-line therapy for osteoporosis in postmenopausal women due to a combination of efficacy, long-term safety data, and low cost due to generic availability in the market. These agents work by decreasing bone resorption by suppressing osteoclast activity resulting in a net gain in bone mass. The most commonly recommended bisphosphonates include alendronate, risedronate, and zoledronic acid due to the broad ability to prevent spinal and hip fractures whereas 
ibandronate prevents spinal fractures only. ${ }^{12-15}$ The medications differ with respect to efficacy due to binding affinity, frequency and route of administration, and overall reduction in fracture risk. In patients with a history of vertebral fracture or osteoporosis of the hip, alendronate therapy reduced the occurrence of spine and hip fractures more than $50 \%$ in 3 years. ${ }^{16,17}$ In patients without a history of vertebral fracture, alendronate reduced the incidence of subsequent fractures by $44 \%$ overall. ${ }^{15}$ In the VERT study, risedronate demonstrated a $41 \%$ to $49 \%$ reduction in vertebral fractures and a $36 \%$ reduction in nonvertebral fractures over 3 years. ${ }^{12}$ Similarly, ibandronate reduces vertebral fracture risk by $50 \%$ over 3 years. To date, ibandronate does not have sufficient evidence for reduction in hip fractures and has not shown impact on risk of nonvertebral fractures.$^{18}$ In the HORIzON trial, zoledronic acid reduced vertebral fracture risk by approximately $70 \%$ and decreased hip and nonvertebral fractures by $35 \%{ }^{14,19}$ In addition to treating osteoporosis, bisphosphonates are FDA approved to treat other skeletal conditions, such as Paget disease, metastatic bone malignancies, and osteogenesis imperfecta. ${ }^{20-22}$

Some advantages to bisphosphonate therapy include flexible dosing options (eg, daily, weekly, monthly, or yearly) and route of administration choice (eg, intravenous [IV] or oral) for patients. Historically, bisphosphonates have been regarded as first-line therapy, but more recently, prescribers have shifted towards basing initial therapy off of risk stratification. For instance, it is appropriate to start with an injectable agent in patients who are classified as very high risk. Patients who qualify for bisphosphonate therapy may be initiated on oral therapy, usually with alendronate or risedronate, and switched to a yearly IV zoledronic acid infusion if contraindications or intolerance to oral therapy exists. Since the optimal treatment duration of bisphosphonate therapy is not well defined, some experts suggest allowing a drug holiday for patients on prolonged therapy after re-evaluating the fracture risk. The AACE/ ACE guidelines indicate a drug holiday may be considered for individuals who have received 5 years of stable oral therapy or 3 years of IV bisphosphonate therapy in high-risk patients. Clinicians may consider a nonbisphosphonate therapy during the drug holiday period in those individuals who are stratified as very high risk. ${ }^{11}$

Most of the bisphosphonates have a strong affinity for forming complexes with minerals such as calcium, magnesium, and aluminumand iron-containing food and beverages that cannot be absorbed. Given the poor rate of absorption when administered orally, it is vital that bisphosphonates are given 30 to 60 minutes prior to eating to ensure maximum bioavailability. Patients must take the medication on an empty stomach with 8 ounces of water to decrease the risk of the medication being lodged in the esophagus and must remain upright for at least 30 minutes to minimize esophageal irritation. Unlike alendronate and ibandronate, risedronate is available as an enteric-coated, delayed release tablet and must be taken immediately after breakfast with 4 ounces of water. Bisphosphonate therapy is contraindicated in patients who are unable to adhere to the administration guidelines or who have esophageal disorders that may delay emptying and transit of the agent..$^{20,22}$ IV bisphosphonate therapy may require pretreatment with acetaminophen or nonsteroidal anti-inflammatory drugs (NSAIDs) in order to minimize influenza-like symptoms associated with acute-phase injection reactions. The risk of infusion-related reactions significantly decreases upon repeated administrations, from $30 \%$ to lower than $7 \% .{ }^{23}$ Prior to starting therapy, serum calcium, vitamin D, and renal function must be evaluated. It is recommended to correct calcium and vitamin D deficiencies prior to initiating therapy with bisphosphonates. ${ }^{20-22}$

Bisphosphonates are generally well tolerated as first-line therapy agents. Adverse effects include upper gastrointestinal discomfort (eg, heartburn, esopagheal ulcers, erosion), abdominal pain, constipation, diarrhea, nausea, and musculoskeletal pain. Osteonecrosis of the jaw (ONJ) is a rare complication causing significant clinical concern that has gained attention over the past several years and, as a result, has led to a decline in bisphosphonate use. The vast majority of ONJ cases have occurred in patients with cancer using high doses of IV bisphosphonates; however, the incidence is much lower for individuals on oral or IV therapy being treated for osteoporosis. ${ }^{24}$ Some additional risk factors for ONJ include high dose and prolonged duration of exposure to bisphosphonate therapy, preexisting dental disease, advanced age, steroid therapy, diabetes, chemotherapy, and female sex. Patients must be advised on the importance of optimizing oral health before starting bisphosphonate treatment. ${ }^{25}$

\section{Calcitonin}

Calcitonin is a polypeptide hormone FDA approved for the treatment of postmenopausal osteoporosis in women greater than 5 years postmenopause. When serum calcium concentrations are elevated, calcitonin is secreted by the thyroid gland. Calcitonin is not widely used for osteoporosis treatment due to less evidence of fracture reduction efficacy compared with alternative therapies. In the PROOF study, nasal calcitonin 200 IU daily reduced new vertebral fractures by $33 \%$ and minimally increased BMD in the spine. However, data demonstrating a reduction in nonvertebral fractures and hip fracture risk in patients treated with calcitonin are lacking. ${ }^{26}$ Calcitonin is available as a subcutaneous injection, intramuscular injection, and generically as a nasal spray. Additional studies are underway for the first oral form of a calcitonin-salmon recombinant to treat osteoporosis. Typically, injectable calcitonin is reserved for patients in whom alternative treatments are not suitable due to either a contraindication or unwillingness to use other medications. The most common adverse effects for the nasal spray dosage form are rhinitis and epistaxis. Nausea, reduced 
appetite, diarrhea, abdominal pain, local inflammatory reactions, and flushing are common adverse effects experienced with the injectable formulations. ${ }^{27}$

\section{Hormonal Therapy (Estrogens)}

Estrogen therapy is only approved for the prevention of osteoporosis in postmenopausal women with significant risk, particularly when nonestrogen medications have been evaluated. It is available in several dosage forms and may be formulated with estrogen, progestin, or a combination of estrogen-progestin. Hormone therapy use has declined drastically after the results of the Women's Health Initiative (WHI) were published; this study evaluated more than 16,000 postmenopausal women with osteoporosis over a mean follow-up of 5.2 years. The findings illustrated a $34 \%$ vertebral and hip fracture reduction; however, significant adverse effects of estrogens limited the overall place in therapy. The benefits of hormone therapy did not outweigh the increased risks of breast cancer, stroke, and venous thromboembolism (VTE) noted in the study. ${ }^{28}$ Some boxed warnings and contraindications to therapy include increased risk of endometrial cancer in women with unopposed estrogens, increased risks of stroke, deep venous thromboembolism (DVT), pulmonary embolism (PE), MI, invasive breast cancer, known or suspected estrogen-dependent neoplasia, and history of breast cancer. ${ }^{29}$ Estrogens have similar efficacy compared with bisphosphonates for the prevention of osteoporosis; however, unlike bisphosphonates, the BMD is rapidly lost upon discontinuation of hormonal therapy.

\section{Selective Estrogen Receptor Modulators (With and Without} Conjugated Equine Estrogens)

Raloxifene is a selective estrogen receptor modulator (SERM) FDA approved for prevention and treatment of postmenopausal osteoporosis; however, it is generally reserved as preventive therapy. SERMs exert a dual effect where BMD is increased through the estrogenic agonist properties and breast and uterine tissue remain unaffected due to the estrogenic antagonist mechanism. Raloxifene is available generically and is administered $60 \mathrm{mg}$ orally once daily. This agent is an alternative to bisphosphonate therapy in women who are at high risk for breast cancer or who are not candidates for bisphosphonates due to an intolerance or contraindication. ${ }^{11}$ Raloxifene decreases the risk of vertebral fractures by $30 \%$ to $68 \%$ and does not have an effect on nonvertebral or hip fractures. ${ }^{30,31}$ Additionally, clinical studies have demonstrated increases in spine and hip BMD, but discontinuation of therapy results in a rapid loss of benefits after 12 to 24 months. ${ }^{11}$

A SERM combination drug containing bazedoxifene and conjugated equine estrogen is approved also for the prevention of postmenopausal osteoporosis. This combination therapy demonstrated an increase in BMD over 24 months at the lumbar spine and hip. Additionally, improvement in vasomotor symptoms, such as hot flashes and vulvo-vaginal atrophy, has been established..$^{32}$ In a 3-year study of healthy postmenopausal women between the ages of 55 and 85 years, bazedoxifene alone showed a reduction in new vertebral fractures with no reduction in risk of nonvertebral fractures. ${ }^{33}$ The SERMs carry the same boxed warnings, contraindications, and precautions as previously noted for estrogen therapy. Adverse effects of SERM agents include hot flashes, VTE, cardiovascular disease, vaginal bleeding, and increased preexisting hypertriglyceridemia. ${ }^{34,35}$ In general, bazedoxifene-conjugated equine estrogen has limited utility given the lack of long-term fracture and safety data compared with raloxifene.

\section{RANK/RANKL Inhibitors}

Denosumab is a monoclonal antibody that prevents the binding of receptor activator of nuclear factor- $\kappa \mathrm{B}$ ligand (RANKL), a transmembrane protein, to its receptor RANK located on the surface of osteoclasts. As a result, preosteoclasts are unable to differentiate into mature cells; the function of existing mature osteoclasts is thus inhibited, leading to reduced bone resorption and increased bone mass. ${ }^{36}$ According to the AACE/ACE guidelines, denosumab has comparable efficacy to bisphosphonates and can be considered at parity with bisphosphonates as a first-line option in patients who are at a high risk for fracture and unable to use oral therapy or in those who are at very high risk of fracture regardless of oral tolerability. In the FREEDOM study, the risks of hip, vertebral, and nonvertebral fractures were reduced by $40 \%, 68 \%$, and $20 \%$, respectively, after 3 years when compared with placebo. ${ }^{37}$ An additional extension phase at the end of 10 years showed an increase in spine BMD (21.7\%) and total hip (9.2\%). ${ }^{38}$

Denosumab is administered as a 60 -mg subcutaneous injection every 6 months by a healthcare professional. Due to the lack of generic availability for denosumab, it may be cost prohibitive for some patients. Patients must be advised to take at least $1000 \mathrm{mg}$ of calcium and 400 units of vitamin D daily. ${ }^{36}$ It is imperative patients remain adherent to the dosing schedule for denosumab because, unlike bisphosphonate therapy, immediate discontinuation may result in a rapid increase in fracture risk. Patients must be evaluated for hypocalcemia, and providers should correct any abnormalities prior to starting denosumab. Warnings and precautions with denosumab therapy include ONJ, atypical femoral fractures, skin infections, dermatitis, rashes, eczema, and over-suppression of bone. Common adverse reactions include back pain, hypercholesterolemia, musculoskeletal pain, and cystitis. ${ }^{36}$

\section{Anabolic Agents}

\section{Parathyroid Hormone and Analogs}

Teriparatide, a recombinant form of parathyroid hormone, and abaloparatide, a synthetic analog of parathyroid-related protein, 
are known as anabolic agents that exert their effects by increasing the formation of bone. Both agents are approved for the treatment of postmenopausal women with osteoporosis with high risk for fracture. ${ }^{39,40}$ Parathyroid hormone and analogs are rarely used as first-line therapy due to a combination of higher cost, daily subcutaneous injections, availability of oral generic low-cost medications, and long-term safety concerns. Teriparatide and abaloparatide decrease the risk of nonvertebral and vertebral fractures and increase hip and spine BMD. ${ }^{41,42}$ In a post hoc analysis of the ACTIVE trial, treatment with abaloparatide significantly decreased vertebral fractures compared with placebo $0.5 \%$ vs $5.6 \% ; P<.001)$, relative risk reduction of $91 \%$, in women with a baseline 10-year risk of major osteoporotic fracture greater than or equal to $10 \%$ or hip fracture greater than or equal to $5 \%$ and was also associated with significantly fewer nonvertebral $(2.7 \%$ vs $5.8 \% ; P=.036)$, major osteoporotic ( $1.3 \%$ vs $6.0 \% ; P<.001)$, and clinical fractures compared with placebo ( $3.5 \%$ vs $8.2 \% ; P=.006)$. The difference in fracture risk reduction between abaloparatide and teriparatide was not statistically significant. ${ }^{43}$ Oftentimes in clinical practice, patients are treated with bisphosphonates after 2 years of therapy with teriparatide or abaloparatide in order to preserve the increase in bone density. ${ }^{44,45}$ If bisphosphonates cannot be used, clinicians may consider the use of raloxifene or denosumab. ${ }^{46,47}$

Abaloparatide must be refrigerated prior to first-time use and may be stored at room temperature for up to 30 days upon opening, whereas teriparatide must be refrigerated at all times, which may be burdensome to patients. ${ }^{39,40}$ Abaloparatide is currently undergoing additional phase 3 studies in a transdermal formulation that may have an advantage over daily injections. ${ }^{48}$ An injection formulation using teriparatide as a reference product was approved through the 505(b)(2) pathway and launched in June 2020 as a potentially lower-cost option for patients. ${ }^{49}$

Use beyond 2 years is not recommended due to the box warning regarding an increased incidence of osteosarcoma noted in rats during clinical studies. Teriparatide should not be used in patients at an increased risk of osteosarcoma, such as those with Paget disease, or pediatric and adult patients with open epiphyses. Notable warnings and precautions for abaloparatide and teriparatide include orthostatic hypotension, hypercalcemia, hypercalciuria, and urolithiasis. Arthralgia, pain, and nausea are common adverse reactions experienced by patients on teriparatide. ${ }^{39}$ Adverse effects of abaloparatide include hypercalciuria, dizziness, headache, palpitations, fatigue, upper abdominal pain, and vertigo. ${ }^{40}$

\section{Sclerostin Inhibitor}

In April 2019, romosozumab-aqqg was approved in the US market for the treatment of osteoporosis in postmenopausal women at high risk for fracture. This monoclonal antibody agent has a unique mechanism of action where it exerts its primary action of anabolic bone formation and a secondary action to decrease bone resorption. ${ }^{50}$ In the FRAME trial, patients were treated with romosozumab for the first 12 months and then denosumab for an additional 12 months. At the end of the first 12 months, vertebral fractures were reduced by $73 \%$ in the romosozumab arm and at the end of 24 months by $75 \%$ in the romosozumab/denosumab arm. The clinical trial did not show a significant decrease in nonvertebral fractures, likely due to the low baseline FRAX 10-year risk in the study population. ${ }^{51}$ In the ARCH study, patients received 12 months of romosozumab or 12 months of alendronate therapy followed by an additional 12 months of alendronate treatment in both arms. Compared with the FRAME study, the ARCH study population had a higher risk for fracture at baseline. The study results showed a significant reduction for hip, nonvertebral, and vertebral fractures by $38 \%, 19 \%$, and $48 \%$, respectively. Furthermore, the ARCH study demonstrated an increase in MI, stroke, and cardiovascular death, which consequently led to the boxed warning. ${ }^{52}$ It is important to note that therapy must not be initiated if the patient has experienced an MI or stroke within the preceding 12 months. Patients must be advised to adequately supplement with calcium and vitamin D. Common adverse reactions with romosuzumab include arthralgia and headache, with additional important warnings for ONJ, atypical femoral fracture, and hypersensitivity reactions, such as rash or angioedema. ${ }^{53}$ Romosozumab is administered as a monthly injection by a healthcare provider for up to 1 year only. After 12 months, patients may be treated with a bisphosphonate or denosumab. ${ }^{11}$

\section{Combination and Sequential Therapy}

The benefit of combined treatment is still an ongoing investigation with minimal data to support its use today. The ES and AACE/ACE guidelines suggest there are not enough data to support fracture risk reduction in most cases. If a patient at high risk of osteoporosis is being treated for menopause with estrogen therapy or breast cancer with raloxifene, it may be appropriate to consider another agent depending on the risk-versus-benefit profile. ${ }^{11}$ Additionally, there are concerns for compounded costs to the patient and adverse effects when using multiple agents. Sequential therapy is starting to gain traction, and there is growing literature indicating it may outperform single therapy when administered for the same time period. ${ }^{54}$ The VERO trial results suggest there are better outcomes when administering an anabolic agent first followed by antiresorptive therapy. ${ }^{55}$ Additional research and data are needed to better define these clinical algorithms.

\section{Economic Burden of Osteoporosis}

There is a significant economic burden on the healthcare system for patients who experience an osteoporosis-related fracture and 
payers managing overall costs. Despite the available treatments and push for preventive measures, in 2015 approximately 2 million Americans experienced 2.3 million osteoporosis-related bone fractures, which in turn led to an increase in hospitalizations and healthcare resource utilization. To compare, these osteoporosisrelated bone fractures accounted for more hospitalizations than MI, stroke, and breast cancer combined. Direct costs for osteoporosis, such as medications, occupational therapies, and inpatient and outpatient visits, and indirect costs, such as loss of productivity, are expected to rise to more than $\$ 95$ billion in $2040 .{ }^{56}$ Generally, hip fractures are the most expensive type of fracture to manage because they may require emergency surgery, add further radiological and laboratory costs, and cause an increased length in hospital stay and potentially long-term care facility stay. Furthermore, hip fractures are linked to a higher mortality, up to $20 \%$ to $24 \%$ compared with other osteoporosis-related fractures. ${ }^{57,58}$ If a patient has a prior history of an osteoporotic fracture, there is a higher likelihood of experiencing another fracture.

A recent retrospective healthcare study in a large payer database examined economic burden in 302,722 patients who experienced an osteoporotic fracture. The study found that $79.4 \%$ patients did not have a diagnosis or treatment on-board for osteoporosis 12 months prior to the fracture. Additionally, 12 months after the occurrence of a fracture, total healthcare costs were estimated to be approximately $\$ 30,000$, of which the patient paid an average of $\$ 3000$. An increase in costs was found with subsequent fractures. The study also concluded the primary areas for osteoporotic fractures were the spine (21.9\%), radius or ulna (19.5\%), and hip (13.7\%), which is consistent with other published literature. ${ }^{59}$ Several opportunities for improvement as it relates driving down healthcare and patient costs can be achieved by ensuring timely bone density testing, implementing recommended lifestyle modifications, and preventing repeat fractures with appropriate treatment. Managed care pharmacists play a critical role in helping patients gain access to appropriate therapy, ensuring medication adherence, and assisting with overall cost reduction.

\section{Managed Care Considerations: Utilization Management Strategies}

In many managed care organizations, the prior authorization (PA) process is intended to ensure treatments are clinically efficacious, safe, and cost-effective for each patient. The PA criteria are created using a combination of published clinical trial data, peer-reviewed medical literature, guideline recommendations, comparativeeffectiveness research, and current standards of care. Criteria are typically shared with an independent external pharmacy and therapeutics (P\&T) committee comprising physicians, pharmacists, and nurses to provide feedback as it relates to real-world practice prior to full implementation. Examples of general PA criteria for higher cost medications from a variety of managed care organizations and health plans are:

- Required medical information: Diagnosis of postmenopausal osteoporosis at a high risk for fracture confirmed by DEXA hip (femoral) or lumbar spine T-score less than or equal to -2.5 or history of T-score between -1.0 and -2.5 if FRAX fracture probability is greater than or equal to $20 \%$ or hip fracture probability is 3\%

- Prerequisite therapy requirements: Documented trial and failure or contraindication to oral bisphosphonate and/or IV bisphosphonate therapy followed by denosumab

- Coverage duration: If requesting abaloparatide or teriparatide, therapy must not exceed a total duration of 24 months (or 12 months in the case of romosozumab)

- Additional information: Submission of chart notes and lab values for clinical review

Another utilization management strategy commonly implemented is step therapy programs. Step therapy programs help to shift prescribing patterns from high-cost generics and brands to lower-cost generics with therapeutic equivalency and safety, resulting in savings for both the patient and payers. ${ }^{60}$ For instance, payers may require a trial and failure of alendronate before shifting to higher-cost bisphosphonates, such as ibandronate, risedronate, or IV zoledronic acid. In addition to PA and step therapy strategies, quantity level limits are often programmed into the adjudication platforms to ensure there is an appropriate supply of medication being dispensed along with an appropriate length of therapy.

\section{The Role of the Managed Care Pharmacist}

Osteoporosis is often regarded as a "silent" condition and many times patients do not understand the importance of medication adherence and lifestyle modifications until a fracture occurs. Unfortunately, adherence to osteoporosis medications is generally suboptimal, which leads to increased direct and indirect costs, reduced efficacy, and increased morbidity and mortality. Common patient barriers to therapy include fear of adverse effects, confusion with extended dosing intervals, and lack of knowledge regarding osteoporosis-related risks and long-term outcomes. ${ }^{61}$ The impact on quality of life plays a large role in patients who experience all types of fractures, particularly hip fractures. It is common for patients to not return to their baseline level of functioning prior to the hip fracture despite efforts of rehabilitation therapy. As a result, this leads to increased healthcare provider visits and costs, loss of independence, and increased risk of mortality.

A systematic review of 25 studies was completed to understand the role pharmacists play in osteoporosis management. Pharmacist-led interventions included education on lifestyle modifications and providing educational pamphlets, comprehensively 
reviewing medications, completing risk assessments, and performing arm DEXA scans to stratify patients based on risk. Researchers concluded calcium intake and targeted screening were improved for patients at high risk of osteoporosis with the intervention of a pharmacist. ${ }^{62}$

Healthcare systems have implemented the concept of a fracture liaison service (FLS), which consists of an interdisciplinary team of pharmacists, physicians, physical therapists, and dietitians to aid in the transition of a patient to the primary care provider after experiencing an osteoporosis-related fracture. In this model, patients receive education on the importance of adequate vitamin $\mathrm{D}$ and calcium intake, a DEXA scan to determine BMD measures and need for further therapy, and a full assessment of fracture and fall risk. In a prospective observational study, implementing an FLS resulted in more patients with hip fractures receiving BMD testing, tailored pharmacologic treatments, and follow-up at a fall and fracture clinic. ${ }^{63}$

Managed care pharmacists can play a significant role to narrow the gaps and serve as a resource for both providers and patients alike. Pharmacists are positioned to be the most accessible healthcare providers and have the most opportunities to engage with patients about their healthcare. It is fundamental to communicate, understand, and preserve patient preferences when making treatment decisions. Pharmacists can use motivational interviewing strategies to appreciate and alleviate patient concerns regarding administration and adverse effects of medications. Counseling points should include the importance of vitamin $\mathrm{D}$ and calcium intake, the benefits of weight-bearing exercises, specific medication storage requirements, administration instructions, and encouragement of DEXA testing when appropriate.

\section{Conclusions}

Osteoporosis is a prevalent condition that can lead to significant pain, loss of independence, high economic burden, and mortality. Prevention strategies include early screening, adequate vitamin $\mathrm{D}$ and calcium intake, regular exercise, limiting alcohol intake, and smoking cessation. Several pharmacologic medications are available for the treatment of postmenopausal osteoporosis in women. There is a general consensus across clinical guidelines supporting the use of bisphosphonates as first-line therapy due to a combination of factors including efficacy, route of administration, and generic availability. In cases where the fracture risk is high and patients are unable to use oral therapy, denosumab may be considered as an alternative first-line agent. The AACE/ ACE guidelines stress the importance of clear communication and shared decision making with the patient. ${ }^{11}$ Pharmacists are in an optimal setting to help alleviate patient concerns on adverse effects, advise on risks of osteoporosis, and identify clinically appropriate treatment options.
Author affiliation: Jeenal Patel, PharmD, BCGP, is the formulary manager at WellDyne, Lakeland, FL.

Funding source: This activity is supported by an educational grant from Radius Health, Inc.

Author disclosure: Dr. Patel has no relevant financial relationships with commercial interests to disclose.

Authorship information: Substantial contributions to the concept and design, drafting of the manuscript, and analysis and interpretation of data.

Address correspondence to: Jpatel@welldyne.com

\section{REFERENCES}

1. Sroga GE, Vashishth D. Effects of bone matrix proteins on fracture and fragility in osteoporosis. Curr Osteoporos Rep. 2012;10(2):141-150. doi: 10.1007/s11914-012-0103-6

2. Sandhu SK, Hampson G. The pathogenesis, diagnosis, investigation and management of osteoporosis. J Clin Pathol. 2011;64(12):1042-1050. doi: 10.1136/jsp.2010.077842

3. Khosla S, Oursler MJ, Monroe DG. Estrogen and the skeleton. Trends Endocrinol Metab. 2012;23(11):576-581. doi: 10.1016/j.tem.2012.03.008

4. Cosman F, de Beur SJ, LeBoff MS, et al; National Osteoporosis Foundation. Clinician's guide to prevention and treatment of osteoporosis [published correction appears in Osteoporos Int. 2015;26(7):2045-

2047]. Osteoporos Int. 2014:25(10):2359-2381. doi: 10.1007/s00198-014-2794-2

5. Nelson HD, Haney EM, Chou R, Dana T, Fu R, Bougatsos C. Screening for Osteoporosis: Systematic Review to Update the 2002 U.S. Preventive Services Task Force Recommendation. Agency for Healthcare Research and Quality (US); July 2010. Evidence Syntheses, №. 77.

6. Siris ES, Adler R, Bilezikian J, et al. The clinical diagnosis of osteoporosis: a position statement from the National Bone Health Alliance Working Group. Osteoporos Int. 2014;25(5):1439-1443. doi: 10.1007/ s00198-014-2655-z

7. Wright NC, Looker AC, Saag KG, et al. The recent prevalence of osteoporosis and low bone mass in the United States based on bone mineral density at the femoral neck or lumbar spine. J Bone Miner Res. 2014;29(11):2520-2526. doi: 10.1002/jbmr.2269

8. Carmona RH. Office of the Surgeon General (US). Bone Health and Osteoporosis: A Report of the Surgeon General. Office of the Surgeon General (US); 2004.

9. Camacho PM, Petak SM, Binkley N, et al. American Association of Clinical Endocrinologists and American College of Endocrinology Clinical Practice Guidelines for the Diagnosis and Treatment of Postmenopausal Osteoporosis - 2016. Endocr Pract. 2016;22(suppl 4):1-42. doi: 10.4158/EP161435.GL 10. Gallagher TC, Geling 0, Comite F. Missed opportunities for prevention of osteoporotic fracture. Arch Intern Med. 2002;162:450-456.

11. Camacho PM, Petak SM, Binkley N, et al. American Association of Endocrinologists/American College of Endocrinology Clinical Practice Guidelines for the Diagnosis and Treatment of Postmenopausal Osteoporosis - 2020 update. Endocr Pract. 2020;26(suppl 1):1-46. doi: 10.415/GL-2020-0524SUPPL 12. Harris ST, Watts NB, Genant HK, et al; Vertebral Efficacy With Risedronate Therapy (VERT) Study Group. Effects of risedronate treatment on vertebral and nonvertebral fractures in women with postmenopausal osteoporosis: a randomized controlled trial. JAMA. 1999;282(14):1344-1352. doi: 10.1001/jama.282.14.1344

13. Reginster J, Minne HW, Sorensen OH, et al; Vertebral Efficacy with Risedronate Therapy (VERT) Study Group. Randomized trial of the effects of risedronate on vertebral fractures in women with established postmenopausal osteoporosis. Osteoporos Int. 2000;11(1):83-91. doi: 10.1007/s001980050010 14. Black DM, Delmas PD, Eastell R, et al; HORIZON Pivotal Fracture Trial. Once-yearly zoledronic acid for treatment of postmenopausal osteoporosis. N Engl J Med. 2007;356(18):1809-1822. doi: 10.1056/ NEJMoa067312

15. Cummings SR, Black DM. Thompson DE, et al. Effect of alendronate on risk of fracture in women with low bone density but without vertebral fractures: results from the Fracture Intervention Trial. JAMA. 1998;280:2077-2082.

16. Black DM, Cummings SR, Karpf DB, et al; Fracture Intervention Trial Research Group. Randomised trial of effect of alendronate on risk of fracture in women with existing vertebral fractures. Lancet. 1996;348(9041):1535-1541. doi: 10.1016/50140-6736(96)07088-2

17. Black DM, Thompson DE, Bauer DC, et al; FIT Research Group. Fracture risk reduction with alendronate in women with osteoporosis: the Fracture Intervention Trial [published correction appears in J Clin Endocrinol Metab. 2001;86(2):938]. J Clin Endocrinol Metab. 2000;85(11):4118-4124. doi: $10.1210 /$ jcem.85.11.6953

18. Chesnut CH 3rd, Skag A, Christiansen C, et al; Oral Ibandronate Osteoporosis Vertebral Fracture Trial in North American and Europe (BONE). Effects of oral ibandronate administered daily or intermittently on fracture risk in postmenopausal osteoporosis. J Bone Miner Res. 2004;19(8):1241-1249. doi: 10.1359/JBMR.040325

19. Lyles KW, Colón-Emeric CS, Magaziner JS, et al; HORIZON Recurrent Fracture Trial. Zoledronic acid and clinical fractures and mortality after hip fracture. N Engl J Med. 2007;357(18):1799-1809. doi: 10.1056/NEJMoa074941

20. Fosamax. Prescribing information. Merck Sharp \& Dohme Corp; 2019; Accessed September 30, 2020. merck.com/product/usa/pi_circulars//ffosamax/fosamax_pi.pdf

21. Actonel. Prescribing information. Procter \& Gamble Pharmaceuticals and sanofi-aventis U.S. LLC; 2009; Accessed September 30, 2020. www.accessdata.fda.gov/drugsatfda_docs/ label/2007/020835s028lbl.pdf

22. Reclast. Prescribing information. Novartis Pharmaceuticals Corporation; 2020; Accessed September 30, 2020. novartis.us/sites/www.novartis.us/files/reclast.pdf?campaign=xjffzswh

23. Ganesan K, Bansal P, Goyal A, et al. Bisphosphonate. In: StatPearls. StatPearls Publishing; 2020. 
24. Khosla S, Burr D, Cauley J, et al. Bisphosphonate-associated osteonecrosis of the jaw: report of a task force of the American Society for Bone and Mineral Research. J Bone Miner Res. 2007;22(10):1479-1491. doi: 10.1359/jbmr.0707onj

25. Khan AA, Morrison A, Hanley DA, et al; International Task Force on Osteonecrosis of the Jaw. Diagnosis and management of osteonecrosis of the jaw: a systematic review and international consensus. J Bone Miner Res. 2015;30(1):3-23. doi: 10.1002/jbmr.2405

26. Chesnut CH 3rd, Silverman S, Andriano K, et al; PROOF Study Group. A randomized trial of nasal spray salmon calcitonin in postmenopausal women with established osteoporosis: the prevent recurrence of osteoporotic fractures study. Am J Med. 2000;109(4):267-276. doi: 10.1016/s0002-9343(00)00490-3

27. Miacalcin. Prescribing information. Novartis; 2017. Accessed November 23, 2020. www.accessdata. fda.gov/drugsatfda_docs/label/2017/020313s036lbl.pdf

28. Rossouw JE, Anderson GL, Prentice RL, et al. Risks and benefits of estrogen plus progestin in healthy postmenopausal women: principal results from the Women's Health Initiative randomized controlled trial. JAMA. 2002;288(3):321-333. doi: 10.1001/jama.288.3.321

29. Prempro. Prescribing information. Wyeth Pharmaceuticals Inc; 2009; Accessed September 30, 2020. www.accessdata.fda.gov/drugsatfda_docs/label/2009/020527s045lbl.pdf

30. Maricic M, Adachi JD, Sarkar S. Wu W. Wong M, Harper KD. Early effects of raloxifene on clinical vertebral fractures at 12 months in postmenopausal women with osteoporosis. Arch Intern Med. 2002;162(10):1140-1143. doi: 10.1001/archinte.162.10.1140

31. Ettinger B, Black DM, Mitlak BH, et al; Multiple Outcomes of Raloxifene Evaluation (MORE) Investigators. Reduction of vertebral fracture risk in postmenopausal women with osteoporosis treated with raloxifene: results from a 3-year randomized clinical trial [published correction appears in JAMA. 1999;282(22):2124]. JAMA. 1999;282(7):637-645. doi: 10.1001/jama.282.7.637

32. Gennari L, Merlotti D, De Paola V, Martini G, Nuti R. Bazedoxifene for the prevention of postmenopausal osteoporosis. Ther Clin Risk Manag. 2008;4(6):1229-1242. doi: 10.2147/tcrm.33476

33. Silverman SL, Christiansen C, Genant HK, et al. Efficacy of bazedoxifene in reducing new vertebral fracture risk in postmenopausal women with osteoporosis: results from a 3-year, randomized, placeboand active-controlled clinical trial. J Bone Miner Res. 2008:23(12):1923-1934. doi: 10.1359/jbmr.080710 34. Duavee. Prescribing information. Wyeth Pharmaceuticals Inc; 2019; Accessed September 30, 2020. labeling.pfizer.com/Showlabeling.aspx?id=1174

35. Evista. Prescribing information. Eli Lilly and Company; 2018; Accessed September 30, 2020. pi.lilly. com/us/evista-pi.pdf

36. Prolia. Prescribing information. Amgen Inc; 2020; Accessed September 30, 2020. pi.amgen.com/ / media/amgen/repositorysites/pi-amgen-com/prolia/prolia pi.pdf

37. Cummings SR, San Martin J, McClung MR, et al; FREEDOM Trial. Denosumab for prevention of fractures in postmenopausal women with osteoporosis [published correction appears in N Engl J Med. 2009;361(19):1914]. N Engl J Med. 2009;361(8):756-765. doi: 10.1056/NEJMoa0809493

38. Bone HG, Wagman RB, Brandi ML, et al. 10 years of denosumab treatment in postmenopausal women with osteoporosis: results from the phase 3 randomised FREEDOM trial and open-label extension. Lancet Diabetes Endocrinol. 2017;5(7):513-523. doi: 10.1016/\$2213-8587(17)30138-9

39. Forteo. Prescribing information. Eli Lilly and Co; 2020; Accessed September 30, 2020. pi.lilly.com/ us/forteo-pi.pdf

40. Tymlos. Prescribing information. Radius Health, Inc: 2020; Accessed September 30, 2020. radiuspharm.com/wp-content/uploads/tymlos/tymlos-prescribing-information.pdf

41. Neer RM, Arnaud CD, Zanchetta JR, et al. Effect of parathyroid hormone (1-34) on fractures and bone mineral density in postmenopausal women with osteoporosis. N Engl J Med. 2001;344(19):1434-1441. doi: 10.1056/NEJM200105103441904

42. Miller PD, Hattersley G, Riis BJ, et al; ACTIV Study Investigators. Effect of abaloparatide vs placebo on new vertebral fractures in postmenopausal women with osteoporosis: a randomized clinical trial [published correction appears in JAMA. 2017;317(4):4422. JAMA. 2016;316(7):722-733. doi: 10.1001/jama.2016.11136 43. McCloskey EV, Fitzpatrick LA, Hu MY, Williams G, Kanis JA. Effect of abaloparatide on vertebral, nonvertebral, major osteoporotic, and clinical fractures in a subset of postmenopausal women at increased risk of fracture by FRAX probability. Arch Osteoporos. 2019;14(1):15. doi: 10.1007/s11657-019-0564-7
44. Black DM, Greenspan SL, Ensrud KE, et al; PaTH Study Investigators. The effects of parathyroid hormone and alendronate alone or in combination in postmenopausal osteoporosis. N Engl J Med. 2003;349(13):1207-1215. doi: 10.1056/NEJMoa031975

45. Rittmaster RS, Bolognese M, Ettinger MP, et al. Enhancement of bone mass in osteoporotic women with parathyroid hormone followed by alendronate. J Clin Endocrinol Metab. 2000;85(6):2129-2134. doi: $10.1210 /$ jcem.85.6.6614

46. Deal C, Omizo M, Schwartz EN, et al. Combination teriparatide and raloxifene therapy for postmenopausal osteoporosis: results from a 6-month double-blind placebo-controlled trial. J Bone Miner Res. 2005;20(11):1905-1911. doi: 10.1359/JBMR.050714

47. Finkelstein JS, Hayes A, Hunzelman JL, Wyland JJ, Lee H, Neer RM. The effects of parathyroid hormone, alendronate, or both in men with osteoporosis. N Engl J Med. 2003;349(13):1216-1226. doi: $10.1056 /$ NEJMoa035725

48. Efficacy \& Safety of Abaloparatide-Solid Microstructured Transdermal System in Postmenopausal Women With Osteoporosis. ClinicalTrials.gov. ClinicalTrials.gov identifier: NCT04064411. Updated August 21. 2019. Accessed November 17, 2020. clinicaltrials.gov/ct2/show/NCT04064411

49. Hagino H, Narita R, Yokoyama Y, Watanabe M, Tomomitsu M. A multicenter, randomized, rater-blinded, parallel-group, phase 3 study to compare the efficacy, safety, and immunogenicity of biosimilar RGB-10 and reference once-daily teriparatide in patients with osteoporosis. Osteoporos Int. 2019;30(10):20272037. doi: 10.1007/s00198-019-05038-y

50. Lim SY, Bolster MB. Profile of romosozumab and its potential in the management of osteoporosis. Drug Des Devel Ther. 2017:11:1221-1231. doi: 10.2147/DDDT.S127568

51. Cosman F, Crittenden DB, Adachi JD, et al. Romosozumab treatment in postmenopausal women with osteoporosis. N Engl J Med. 2016;375(16):1532-1543. doi: 10.1056/NEJMoa1607948

52. Saag KG, Petersen J, Brandi ML, et al. Romosozumab or alendronate for fracture prevention in women with osteoporosis. N Engl J Med. 2017:377(15):1417-1427. doi: 10.1056/NEJMoa1708322

53. Evenity. Prescribing information. Amgen Inc; 2020; Accessed September 30, 2020. pi.amgen.com/ / media/amgen/repositorysites/pi-amgen-com/evenity/evenity_pi hcp_english.ashx

54. Lou S, Lv H, Wang G, et al. The effect of sequential therapy for postmenopausal women with osteoporosis: a PRISMA-compliant meta-analysis of randomized controlled trials. Medicine (Baltimore). 2016:95(49)::55496. doi: 10.1097/MD.0000000000005496

55. Kendler DL, Marin F, Zerbini CAF, et al. Effects of teriparatide and risedronate on new fractures in post-menopausal women with severe osteoporosis (VERO): a multicentre, double-blind, double-dummy, randomised controlled trial [published correction appears in Lancet. 2018;392(10162):2352]. Lancet. 2018:391(10117):230-240. doi: 10.1016/S0140-6736(17)32137-2

56. Lewiecki EM, Ortendahl JD, Vanderpuye-Orgle J, et al. Healthcare policy changes in osteoporosis can improve outcomes and reduce costs in the United States. JBMR Plus. 2019;3(9):e10192. doi: $10.1002 / j \mathrm{jm} 4.10192$

57. Cooper C, Atkinson EJ, Jacobsen SJ, O'Fallon WM, Melton LJ 3rd. Population-based study of survival after osteoporotic fractures. Am J Epidemiol. 1993;137(9):1001-1005. doi: 10.1093/oxfordjournals.aje.a116756 58. Leibson CL, Tosteson AN, Gabriel SE, Ransom JE, Melton LJ. Mortality, disability, and nursing home use for persons with and without hip fracture: a population-based study. J Am Geriatr Soc. 2002:50(10):1644-1650. doi: 10.1046/j.1532-5415.2002.50455.x

59. Williams SA, Chastek B, Sundquist $K$, et al. Economic burden of osteoporotic fractures in US managed care enrollees. Am J Manag Care. 2020;26(5):e142-e149. doi: 10.37765/ajmc.2020.43156 60. Managed care pharmacy 101. Academy of Managed Care Pharmacy (AMCP). Accessed September 30, 2020. amcp.org/about/managed-care-pharmacy-101\#MCP

61. Alami S, Hervouet L, Poiraudeau S, Briot K, Roux C. Barriers to effective postmenopausal osteoporosis treatment: a qualitative study of patients' and practitioners' views. PLoS One. 2016;11(6):e0158365. doi: $10.1371 /$ journal.pone. 0158365

62. Elias MN, Burden AM, Cadarette SM. The impact of pharmacist interventions on osteoporosis management: a systematic review. Osteoporos Int. 2011;22(10):2587-2596. doi: 10.1007/s00198-011-1661-7 63. Ruggiero C. Zampi E, Rinonapoli G, et al. Fracture prevention service to bridge the osteoporosis care gap. Clin Interv Aging. 2015;10:1035-1042. doi: 10.2147/CIA.S76695 


\section{Economic Implications of Osteoporotic Fractures in Postmenopausal Women}

Release date: November 30, 2020

Expiration date: November 30, 2021

Pharmacy Credit

Instructions for Receiving Continuing Pharmacy Education (CPE) Credit: Testing Information

This lesson is free online; request your CE credit at www.PharmacyTimes.org.

\section{Testing Directions}

1. Each participant evaluating the activity is eligible to receive CE credit.

2. To receive your credit online, go to www.PharmacyTimes.org and complete the online posttest and the online activity evaluation form before the expiration date. Your CE credit will be automatically uploaded to CPE Monitor. Please ensure that your Pharmacy Times ${ }^{\circledR}$ account is updated with your NABP e-profile ID number and your date of birth (MMDD format). Participation data will not be uploaded into CPE Monitor if you do not have your NABP e-profile ID number and date of birth entered into your profile on www.PharmacyTimes.org.

4. A 60-year-old postmenopausal woman with history of esophageal strictures fell from standing and sustained a hip fracture 4 months ago. She is now status postsurgical repair and presents to clinic for follow-up and optimization of fracture prevention. Recent lab results indicate the serum calcium and vitamin $D$ are within normal limits. Which of the following is the best treatment option?
A. Alendronate
B. Calcitonin
C. Hormone replacement therapy
D. Zoledronic acid

5. Which of the following would be the best option in order to preserve the increase in bone mineral density after a patient has been treated with abaloparatide for $\mathbf{2}$ years?

3. Which bisphosphonate lacks data to support hip fracture reduction?
A. Alendronate
B. Ibandronate
C. Risedronate
D. Zoledronic acid
A. Alendronate
B. Calcitonin
C. Raloxifene
D. Teriparatide

6. According the post hoc analysis of the ACTIVE trial, what is the relative risk reduction of vertebral fractures in women with a baseline 10-year risk of major osteoporotic fracture greater than $10 \%$ after using abaloparatide compared with placebo?
A. $55 \%$
B. $77 \%$
C. $83 \%$
D. $91 \%$ 


\title{
SUPPLEMENT POLICY STATEMENT
}

\author{
Standards for Supplements to The American Journal of Managed Care ${ }^{\circledR}$
}

All supplements to The American Journal of Managed Care ${ }^{\circledR}$ are designed to facilitate and enhance ongoing medical education in various therapeutic disciplines. All Journal supplements adhere to standards of fairness and objectivity, as outlined below. Supplements to The American Journal of Managed Care ${ }^{\circledR}$ will:

I. Be reviewed by at least 1 independent expert from a recognized academic medical institution.

II. Disclose the source of funding in at least 1 prominent place.

III. Disclose any existence of financial interests of supplement contributors to the funding organization.

IV. Use generic drug names only, except as needed to differentiate between therapies of similar class and indication.

V. Be up-to-date, reflecting the current las of date of publication) standard of care.

VI. Be visually distinct from The American Journal of Managed Care ${ }^{\circledR}$.

VII. Publish information that is substantially different in form and content from that of the accompanying edition of The American Journal of Managed Care ${ }^{\circledR}$.

VIII. Prohibit excessive remuneration for contributors and reviewers.

IX. Carry no advertising.

Publisher's Note: The opinions expressed in this supplement are those of the authors, presenters, and/or panelists and are not attributable to the sponsor or the publisher, editor, or editorial board of The American Journal of Managed Care ${ }^{\circledR}$. Clinical judgment must guide each professional in weighing the benefits of treatment against the risk of toxicity. Dosages, indications, and methods of use for products referred to in this supplement are not necessarily the same as indicated in the package insert for the product and may reflect the clinical experience of the authors, presenters, and/or panelists or may be derived from the professional literature or other clinical sources. Consult complete prescribing information before administering. 
